Upcoming: August 17-21 XVII International Materials Research Congress 2008, VII Congress of Nace International - Section México, M-MRS; August 20-24 5th Intl. Conf. on Hot-Wire Chemical Vapor Deposition, Endorsed; August 31-3 Electroceramics XI, Endorsed; September 15-19 E-MRS 2008 Fall Meeting, E-MRS; September 23-25 AMIC 2008, Endorsed; September 28-2 SBPMat 2008, Endorsed, Brazil-MRS.

\begin{abstract}
To list an event in the Calendar, contact J. Meiksin, Materials Research Society, 506 Keyston Drive, Warrendale, PA 150867573; 724-779-3004 ext. 522; fax 724-779-8313; bulletin@mrs.org.
\end{abstract}

MRS, A-MRS, B-MRS, C-MRS, E-MRS, MRS-A, MRS-I, MRS-J, MRS-K, MRS-R, MRS-S, MRS-T, M-MRS, or IUMRS at the end of an entry indicates sponsorship or cosponsorship of an event by the International Union of Materials Research Societies or one of its adhering bodies. "Endorsed" identifies events endorsed by MRS.

$\nabla$ identifies a new or revised entry this month.

See the May 2008 MRS BULLETIN for July 2008 Calendar entries. For updates, access www.mrs.org.

\section{AUGUST 2008}

3-8 Ultra-High Temperature Ceramics: Materials for Extreme Environment Applications, Lake Tahoe,CA. E-mail info@poly.edu; www.engconfintl.org/8ah.html.

4-8 $\nabla$ Renewable Energy Capturing the Sun, Cambridge, MA MIT Professional Institute, 77 Massachusetts Ave., Cambridge, MA 02139; 617-253-6128; fax 617-2588831; e-mail professionalinstitute@ mit.edu; web.mit.edu/mitpep/pi/ courses/renewable_energy_sun.html.

6-8 8th Annual Conf. on Residual Stresses (ICRS-8), Denver, CO. E-mail flaherty@icdd. com; www.dxcicdd.com/icrs/ default.htm.

10-13 $\nabla$ U.S.-Japan POLYMAT 2008 Summit, Ventura Beach, CA Lesia Linkous, 540-231-3052 e-mail lesiar@vt.edu; www.polyacs.net.

10-15 20th Intl. Conf. on the Application of Accelerators in Research and Industry (CAARI 2008), Fort Worth, TX

CAARI, 940-565-3250; e-mail caari@unt.edu; www.caari.com.

12-14 $\nabla$ IMAPS Photovoltaics Fuel Cells, and Other Emerging Technologies in the Development of Alternative Energy, Albuquerque, NM. IMAPS Headquarters Staff, 611 2nd St., NE, Washington, DC 20002; 202-548-4001; fax 202-548-6115; e-mail imaps@imaps.org; www.imaps.org/energy.
17-21 XVII Internationa Materials Research Congress 2008, VII Congress of Nace International - Section México Cancún, México. http://imrc2008.com. M-MRS.

20-24 5th Intl. Conf. on HotWire Chemical Vapor Deposition Cambridge, MA. E-mail hwcvd5-www@mit.edu; web.mit. edu/hwcvd5. Endorsed.

31-3 Electroceramics XI Manchester, UK. Naomi Hyde, Electroceramics XI, Grosvenor St. Manchester, UK; 44-01613062258; fax 44-0161-3068840; e-mail naomi.hyde@manchester. ac.uk; www.electroceramics11. co.uk/index.htm. Endorsed.

\section{SEPTEMBER 2008}

7-11 5th Intl. Conf. on Fracture of Composites, Polymers and Adhesives, Les Diablerets, Switzerland. Claire Norris, European Structural Integrity SocietyTechnical Committee; e-mail tc4-conference@elsevier.com; www.tc4pa.elsevier.com

7-11 7th European Conf. on Silicon Carbide and Related Materials, Barcelona, Spain. Miguel Vellvehi, e-mail ecscrm2008@ reunionsciencia.es; www.ecscrm08.com.

7-11 Intl. Mesostructured Material Association (IMMA) Belgium. IMMA, C/O Hospitalité Québec, 580, Grande Allée est, Ste. 305, QC, G1R 2K2, Canada 418-522-8182; fax 418-529-7548; e-mail conference@hospitalite. com; hospqbc.forest.net/Clients/ IMMA/imma home.html.

8-10 Micro Nano Breakthrough Conf. (MNBC), Vancouver, WA. Robert D. Rung, Oregon Nanoscience and Microtechnologies Institute, P.0. Box 2041, Corvallis OR 97339; 541-713-1331; fax 541 737-1805; e-mail skip@onami.us oregonstate.edu/conferences/MNBC.

8-12 PolyFilm 2008, Sheffield UK. E-mail j.I.hopkin@sheffield ac.uk; www.polyfilm.eu.com/ conference/polyfilm2008_1.pdf.

8-12 Yucomat 2008 Herceg Novi, Montenegro. E-mail yumrs@itn.sanu.ac.yu.

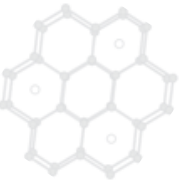

11-13 Society for Biomaterials 2008-Translational Biomaterial Research, Advancing Discoveries from the Laboratory to the Clinic (SFB 2008), Atlanta, GA. Headquarters Office, 15000 Commerce Pkwy., Ste. C, Mt. Laurel, NJ 08054; 856-439-0826; fax 856-439-0525; e-mail info@biomaterials.org; www. biomaterials.org/Meetings/ 08AnnualMeeting/index.cfm.

14-18 Superalloys, Champion, $P A$. TMS, TMS Meetings Services, 184 Thorn Hill Road, Warrendale, PA, 15086; 724-776-9000 x243; fax 724-776-3770; e-mail mtgserv@ tms.org; www.tms.org/Meetings.

15-19 XVII Intl. Symp. on Gas Flow and Chemical Lasers and HighPower Laser Conf. (GCL/HPL 2008), Lisbon, Portugal. Alexandra Jacques; e-mail a.jacques@ist.ut..pt.

15-19 E-MRS 2008 Fall Meeting, Warsaw, Poland. Fall 2008 E-MRS, e-mail warsaw.fall@ e-mrs.org; www.e-mrs.org/ meetings/fall2008. E-MRS.

22-26 Intl. Conf. on Aluminum Alloys (ICAA 11), Aachen, Germany. E-mail icaa11@dgm.de; www.dgm. de/dgm/icaa11.

23-25 Advanced Metallization Conf. (AMC) 2008, San Diego, CA. Jenny BlackDeer, Univ. of California, Berkeley, 1995 University Ave., Berkeley, CA 94720-7010; 510-642-4151; fax

510-642-6027; e-mail

jlb@unex.berkeley.edu; www.unex.berkley.edu/eng/metal. Endorsed.

24-26 $\boldsymbol{\nabla} 2008$ Intl. Conf. on Solid State Devices and Materials, Tsukuba, Japan. Inter Group Corp. Secretariat SSDM, Toranomon Takagi Bldg., 1-7-2, Nishi-shinbashi, Minato-ku, Tokyo, Japan; 81-3-35971108; fax 81-3-3597-1097;

e-mail ssdm@intergroup.co.jp; www.ssdm.jp.

28-2 The Federation of Analytical Chemistry and Spectroscopy Societies (FACSS) 2008, Reno, NV. E-mail gary.

brewer@us.abb.com.

28-2 SBPMat 2008, Guaruja, Brazil.E-mail sbpmat@sbpmat. org.br; www.sbpmat.org.br. Endorsed, Brazil-MRS.

29-1 Euro PM2008, Mannheim, Germany. EPMA, Old Bank Buildings, Bellstone, Shrewsbury, UK : 44-1743-248899; fax 44-1743362968; e-mail fh@epma.com; www.epma.com/pm_2008/home.htm.

\section{OCTOBER 2008}

5-9 Materials Science and Technology 2008 (MS\&T'08), Pittsburgh, PA. Matthew Merwin, US Steel Research and Technology Center, 412-433-7187; e-mail mmerwin@uss.com; mst08. abstractcentral.com.

6-10 ₹ Intl. Workshop Nitride Semiconductors 2008, Montreux, Switzerland. IWN 2008 iwn2008.epfl.ch.

7-8 22nd Intl. Activated Carbon Conf., Pittsburgh, PA. PACS Inc., 409 Meade Dr., Coraopolis, PA 15108; 724-457-6576; fax 724457-1214; e-mail Barb@pacslabs. com; pacslabs.com/conferences/ iacc/iacc22.php.

12-17 ECS Meeting Fall 2008 Honolulu, HI. The Electrochemical Society, ECS, 65 S. Main Street, Building D, Pennington, NJ 085342839; 609-737-1092; fax 609-7372743; e-mail ecs@electrochem.org; www.electrochem.org/meetings.

19-24 $\nabla$ American Vacuum Society (AVS) 55th Intl. Symp and Exposition, Boston, MA. 110 Yellostone Dr., Suite 120, Chico, CA 95973: 530-896-0477; fax 530896-0487; e-mail della@avs.org www.avs.org.

20-23 Intl. Congress on Applications of Lasers and Electro-Optics (ICALEO) 2008, Temecula, CA. E-mail bcohen@ laserinstitute.org; www.icaleo.org.

22-26 2nd Intl. Symp. on Transparent Conducting Oxides, Crete, Greece. MITOS S.P. e-mail info@mitos.com.gr; www.mitos. com.gr. Endorsed.

27-30 $\nabla$ 5th Intl. Congress of Nano-Bio and Clean Tech 2008 San Francisco, CA. International Association of Nanotechnology, Inc 2386 Fair Oaks Blvd. Sacramento, CA 95825; 916-529 4119; fax 916-421-6366; e-mail info@ianano.org; www.ianano. org/CallforPapers.htm.

27-31 2008 Fuel Cell Seminar and Exposition, Phoenix, AZ. 202973-8671; fax 202-331-0111; e-mail fuelcell@courtesyassoc.com; fcse.confex.com/fcse/2008/cfp.cgi.

\section{NOVEMBER 2008}

2-6 41st Intl. Symp. on Microelectronics, Providence, $R$ Jackki Morris-Joyner, e-mail jmorris@imaps.org; www.imaps. org/imaps2008/index.htm.

10-14 53rd Conf on Magnetism and Magnetic Materials (MMM), Austin, TX. Dieter Weller, e-mail dieter.weller@seagate.com; www.magnetism.org.

13-14 Intl. Conf. on Automotive Technology (ICAT 2008), Istanbul, Turkey. www.icatconf.org.

17-20 $\nabla$ 14th Intl. Conf. on Thin Films and Reactive Sputter Deposition 2008 (ICTF14)

Ghent, Belgium. University of Ghent, Ghent, Europe, Belgium e-mail wouter.leroy@ugent.be; www.ictf14.ugent.be.

17-21 APS Annual DPP Meeting, Dallas, TX. APS, www.aps.org/meet

25-28 Unifying Concepts in Glass Physics IV (UCGP2008), Kyoto, Japan. E-mail ucgp08-qa@ cheme.kyoto-u.ac.jp; www-tph. cheme.kyoto-u.ac.jp/UCGP2008.

\title{
DECEMBER 2008
}

1-5 MRS Fall Meeting Boston, MA. Materials Research Society, 506 Keystone Drive, Warrendale, PA 15086; 724-779-3003; fax 724-779-8313 


\section{JANUARY 2009}

20-22 7th Intl. Conf, on Composite Science and Technology, Sharjah, United Arab Emirates. E-mail iccst7@aus.edu; www.aus. edu/conferences/iccst7

24-28 $\nabla$ LabAutomation2009, Palm Springs, CA. Association for Laboratory Automation, 330 West State St., Geneva, IL 60134; 888-733-1252; fax 630-578-0172; e-mail bdreier@labautomation.org; www.labautomation.org/LA09.

\section{MARCH 2009}

16-20 APS March Meeting 2009, Pittsburgh, PA. APS www.aps.org/meet.

17-20 Microscopy of Semiconducting Materials 2009 Oxford, UK. E-mail Victoria@rms org; www.rms.org.uk. Endorsed.

\section{APRIL 2009}

13-17 MRS Spring Meeting San Francisco, CA. Materials Research Society, 506 Keystone Drive, Warrendale, PA 15086; 724-779-3003; fax 724-779-8313; e-mailinfo@mrs.org; www.mrs.org. MRS
17-22 Experimental Biology 2009 Annual Meeting, New Orleans, LA. FASEB/OSMC Jean Lash, 9650 Rockville Pike Bethesda, MD, 20814; 301-6347009; fax 301-634-7014; e-mail jlash@faseb.org; www.faseb.org/meetings.

\section{MAY 2009}

2-5 APS April Meeting 2009, Denver, CO. APS, www.aps.org/ meet/calendar.cfm.

\section{6-9 6th Intl. Conf. on High} Temperature Capillarity (HTC 2009), Athens, Greece. Simeon Agathopoulos, Materials Science and Engineering Department, University of Ioannina, GR-451 10, Ioannina, Greece; 30-2651097381; fax 30-265109-7074; e-mail sagat@cc.uoi.gr; www.htc2009. org. Endorsed.

11-15 ACHEMA 2009 Frankfurt, Germany. E-mail janik@ dechema.de; www.achema. de/en/ACHEMA.html.

24-29 Electrochemical Society Meeting Spring 2009 (ECS),

San Francisco, CA. ECS,

65 S. Main St., Bldg. D,

Pennington, NJ, 08534-2839

609-737-1092; fax 609-737-2743

e-mail ecs@electrochem.org;

www.electrochem.org/meetings/

biannual/fut_mtgs.htm.

\section{JUNE 2009}

7-12 $\nabla 21$ st North American Catalysis Society Meeting (NAM), San Francisco, CA. E-mail nam21-info@pnl.gov; www.21nam.org.

\section{8-12 E-MRS 2009 Spring} Meeting, Strasbourg, France. www.emrs-strasbourg.com. E-MRS.

29-2 3rd Intl. Exhibition of the Plastics Industry (PLASTICOS '09), Buenos Aires, Argentina. E-mail info@banpaku.com.ar; www.banpaku.com.ar/ banpaku en.htm.

28-3 ICMAT: Intl. Conf. on Materials for Advanced

Technologies, Singapore. http://mrs.org.sg/icmat2009. MRS-S.

\section{JULY 2009}

9-10 23rd Intl. Activated Carbon Conf., Johannesburg. South Africa. PACS Inc.

409 Meade Dr., Coraopolis, PA 15108; 724-457-6576; fax 724457-1214; e-mail Barb@pacslabs. com; pacslabs.com/conferences/ index.php.
13-18 XIV Intl. Conf. on Small-Angle Scattering (SAS-2009) Oxford, UK. E-mail s.m.king@rl.ac.uk; www.isis.rl.ac.uk/largescale/loq/ SAS2009/SAS2009.htm.

20-25 SBPMat Brazil-MRS, IUMRS-ICAM, Rio de Janeiro, Brazil. E-mail sbpmat@sbpmat. org.br; www.sbpmat.org.br.

Endorsed, Brazil-MRS, IUMRS

\section{OCTOBER 2009}

4-9 Electrochemical Society Meeting Fall 2009 (ECS), Vienna Austria. ECS, 65 S. Main St., Bldg. D, Pennington, NJ, 08534-2839; 609-737-1092; fax 609-737-2743. e-mail ecs@electrochem.org; www.electrochem.org/meetings/ biannual/fut_mtgs.htm.

8-9 24th Intl. Activated Carbon Conf., Pittsburgh, PA. PACS Inc.

409 Meade Dr. Coraopolis, PA

15108; 724-457-6576; fax 724-4571214; e-mail Barb@pacslabs.com; pacslabs.com/conferences/index.php.
18-22 The Federation of Analytical Chemistry and Spectroscopy Societies (FACSS)

2009, Louisville, KY.

E-mail ramttidb@yahoo.com

\section{NOVEMBER 2009}

2-6 APS Annual DPP Meeting Atlanta, GA. APS, www.aps.org/ meet/calendar.cfm.

30-4 2009 MRS Fall Meeting, Boston, MA. Materials Research Society, 506 Keystone Dr., Warrendale, PA 15086;

724-779-3003; fax 724-779-8313; e-mail info@mrs.org; www.mrs. org. MRS.

\section{MARCH 2010}

15-19 APS March Meeting 2010, Portland, OR www aps. org/meet/calendar.cfm.

\section{APRIL 2010}

5-9 2010 MRS Spring Meeting, San Francisco, CA. Materials Research Society, 506 Keystone Drive, Warrendale, PA 15086; 724-779-3003; fax 724-779-8313; e-mail info@mrs.org; www.mrs.org. MRS.

\section{NOVEMBER 2010}

29-3 2010 MRS Fall Meeting, Boston, MA. Materials Research Society, 506 Keystone Drive, Warrendale, PA 15086; 724-7793003; fax 724-779-8313; e-mail info@mrs.org; www.mrs.org. MRS.

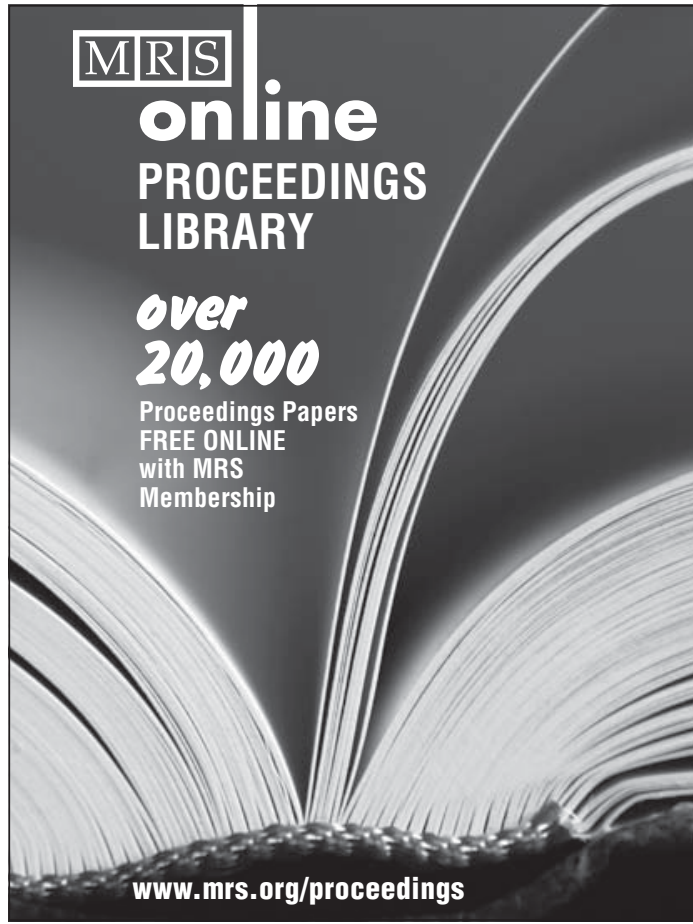

\section{$\mathrm{M} R \mathrm{R}$}

Materials Research Society www.mrs.org

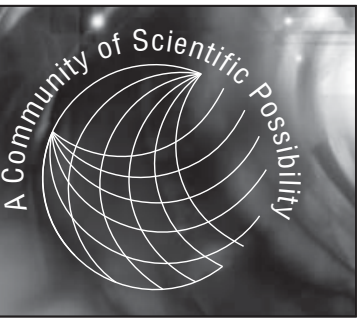

\section{Advertisers in This Issue}

Page No.

\begin{tabular}{lr}
\hline Aldrich/Sigma-Aldrich & Inside back cover \\
\hline High Voltage Engineering Europa B.V. & Inside front cover \\
\hline Hindawi Publishing Corp. & 648 \\
\hline Huntington Mechanical Laboratories, Inc. & Outside back cover \\
\hline Janis Research Company, Inc. & 645 \\
\hline Lake Shore Cryotronics, Inc. & 647 \\
\hline J.A. Woollam Company, Inc. & 646 \\
\hline
\end{tabular}

For free information about the products and services offered in this issue, check http://www.mrs.org/bulletin_ads. 